\section{Heparininduzierte Thrombo- zytopenie Typ II als Ursache eines schweren Rezidivs einer Lungenembolie}

F. Nickels ${ }^{1}$, T. Müller ${ }^{1}$, C. Schulz 1 , C. Nabel ${ }^{1}$, A. Sigel ${ }^{1}$, W. Notheis ${ }^{1}$, F. Muders ${ }^{1}$, M. Reng ${ }^{2}$, G. A. J. Riegger ${ }^{1}$, M. Pfeifer ${ }^{1}$

${ }^{1}$ Klinik und Poliklinik für Innere Medizin II

(Direktor: Prof. Dr. G. A. J. Riegger), Bereich Intensivmedizin, Klinikum der Universität Regensburg

${ }^{2}$ Klinik und Poliklinik für Innere Medizin I (Direktor: Prof. Dr. J. Schölmerich), Klinikum der Universität Regensburg
Zusammenfassung: Die heparininduzierte Thrombozytopenie (HIT) stellt eine seltene Komplikation einer Heparintherapie dar. Die schwere Form HIT II ist gekennzeichnet durch eine Thrombozytopenie und thrombotische und thromboembolische Ereignisse. Wir berichten über einen 24-jährigen Mann, der initial auf unserer Intensivstation mit einer zentralen, rechtsseitigen Lungenembolie und einer Beinvenenthrombose der rechten $\mathrm{V}$. femoralis superficialis aufgenommen, und anschließend einer systemischen Urokinase-Lysetherapie über 7 Tage zugeführt wurde. Darunter kam es zu einer fast vollständigen Rückbildung der pulmonalen Emboli. Anschließend erfolgte die Verlegung des Patienten auf eine periphere Station, wo eine Heparintherapie und eine überlappende Marcumartherapie weitergeführt wurde. Nach 6 Tagen wurde die Wiederaufnahme des Patienten auf die Intensivstation erforderlich. Der Patient bot hierbei das klinische Bild einer hämodynamisch wirksamen, im CT-Thorax bestätigten, beidseitigen zentralen Lungenembolie. Weiterhin zeigte sich eine Zunahme der Beinvenenthrombose rechtsseitig bis in Höhe der V. iliaca externa. Auffallend war zudem, dass die Thrombozytenzahl, die zuletzt vor 3 Tagen kontrolliert wurde, von 112 000/ $\mu$ l auf nun $35000 / \mu \mathrm{l}$ abgefallen war. Klinisch bestand der Verdacht auf eine HIT II. Mittels ELISA konnte der positive Nachweis von Antikörpern gegen PF4-Heparin-Komplexe erbracht werden. Nach Implantation eines temporären V.-cava-Schirmes und unter erneuter Lysetherapie und Umstellung der Heparintherapie auf Lepirudin stabilisierte sich der klinische Zustand mit fast vollständiger Normalisierung der pulmonalen Druckwerte. Die Thrombozytenzahl normalisierte sich bereits innerhalb von 3 Tagen unter Lepirudintherapie. Die HIT II stellt eine schwere Komplikation einer Heparintherapie dar. Neben der Darstellung der Kasuistik wird auf die Früherkennung, DifferenzialDiagnosen, insbesondere zur HIT I und die Therapie eingegangen.

Recurrence of Massive Pulmonary Thromboembolism Caused by Heparin-induced Thrombocytopenia Type II: Heparin-induced thrombocytopenia (HIT) is a rare complication of anticoagulative heparin therapy. The more severe HIT type II is defined by peripheral thrombocytopenia combined with thrombotic and thromboembolic events. We report the case of a 24 year old male patient who was admitted to our ICU with thromboembolic obstruction of the right central pulmonary ar-

Pneumologie 2001; 55: 44-50

(c) Georg Thieme Verlag Stuttgart · New York ISSN 0934-8387 tery, and deep venous thrombosis (DVT) of the right superficial femoral vein. Systemic thrombolytic therapy with urokinase for seven days resulted in nearly complete resolution of the thromboembolic material in the pulmonary arteries. Antithrombotic therapy with intravenous heparin and overlapping oral phenoprocoumon was continued on the regular ward. Six days later, the patient had to be readmitted to the ICU with evidence of hemodynamic compromise due to massive bilateral pulmonary thromboembolism that could be confirmed by CT scan- DVT had extended to the right iliacal vein. Additionally, peripheral thrombocyte counts had markedly declined from 112.000 to $35.000 / \mu \mathrm{l}$ within 3 days, indicating the presence of a Hit type II. This was verified by positive ELISA testing for antibodies against platelet factor 4 (PF4)-heparin-complex. A filter device was temporarilly implanted into the inferior vena cava. The patients condition stabilized upon reinitiated systemic thrombolysis and replacement of heparin therapy against recombinant hirudin. Pulmonary artery pressures normalized. Peripheral thrombocytopenia diminished within three days. HIT type II is a severe complication of anticoagulative therapy with heparin. Here we report a case, and discuss diagnostic procedures as well as differential diagnosis to HIT type I.

\section{Einleitung}

Die heparininduzierte Thrombozytopenie Typ II (HIT II) als seltene, jedoch potenziell lebensgefährliche Komplikation unter Heparintherapie ist gekennzeichnet durch das Leitsymptom der Thrombozytopenie und neu auftretende thrombotische oder thromboembolische Gefäßverschlüssen. Wir berichten über einen 24-jährigen Patienten, der nach primär erfolgreicher Therapie einer Lungenembolie mit Urokinaselyse unter systemischer Heparingabe ein schweres, hämodynamisch wirksames Lungenembolierezidiv bei manifester HIT II erlitt.

\section{Fallbeschreibung}

\section{Anamnese}

Ein 24-jähriger männlicher Patient stellte sich in der Notaufnahme unseres Klinikums vor und berichtete über einen seit ca. 1 Woche bestehenden trockenen Reizhusten und eine progrediente Schwellung des rechten Beines. In der Woche zuvor habe er längere Autofahrten unternommen. In der vergangenen Nacht seien nun vermehrt Reizhusten und seit den frühen Morgenstunden eine zunehmende Atemnot in 
Ruhe und ein Spannungsgefühl im gesamten rechten Bein und der rechten Leiste aufgetreten.

Vorerkrankungen: Z.n. Pneumonie vor 2 Jahren, Z.n. Außenbandruptur des linken Außenknöchels vor 5 Jahren, konservativ versorgt, keine Voroperationen, keine stationären Aufenthalte. Kein Nikotin.

Beruf: Zeitsoldat bei der Bundeswehr, überwiegende Schreibtischtätigkeit.

Familie: Eltern und 2 Brüder, gesund. Großmutter an Lungenembolie verstorben.

\section{Klinischer Aufnahmebefund}

Bei Aufnahme sahen wir einen 24-jährigen Patienten im reduzierten Allgemeinzustand, Größe $175 \mathrm{~cm}$, Gewicht $90 \mathrm{~kg}$, mit auffälliger Ruhedyspnoe und Tachypnoe, die Atemfrequenz betrug 32/min. Zudem fiel eine Lippenzyanose auf. Der Blutdruck wurde mit $150 / 80 \mathrm{mmHg}$ gemessen, die Herzfrequenz betrug 126 Schläge/min. Die Halsvenen waren in halbsitzender Position leicht gestaut ohne Hinweise auf eine Struma. Die Lungen zeigten sich perkutorisch und auskultatorisch unauffällig, ein Pleurareiben war nicht zu verzeichnen. Die Auskultation des Herzens ergab reine Herztöne bei einem tachykarden und regelmäßigen Grundrhythmus. Das Abdomen war palpatorisch weich und ohne Druckschmerz, mit regelrechter Darmperistaltik. Auffallend war eine Schwellung des gesamten rechten Beines bis zur Leiste ohne manifeste Rötung. Es bestand kein Wadendruckschmerz und auch kein Fußsohlendruckschmerz, jedoch wurden Schmerzen bei Palpation der rechten Leiste angegeben. Das linke Bein zeigte einen unauffälligen Befund. Die neurologische Untersuchung ergab einen Normalbefund.

\section{Klinisch-chemische Befunde}

Laborchemisch zeigte sich eine Leukozytose von 16 000/1 mit 83,5\% Neutrophilen im Differenzialblutbild, die Thrombozytenanzahl befand sich bei $136000 / \mu$, das CRP betrug $25 \mathrm{mg} / \mathrm{dl}$ und die LDH $250 \mathrm{U} / \mathrm{l}$. Gerinnungsphysiologisch fielen erhöhte D-Dimere mit 4,5 $\mu \mathrm{g} / \mathrm{ml}$ auf (D-Dimer-Test der Firma DADE-Industries, Normbereich $<0,5 \mu \mathrm{g} / \mathrm{ml})$. Die übrigen bestimmten Gerinnungsparameter wie Quick, PTT, Thrombinzeit und AT III befanden sich im Normbereich. Ebenso fanden sich Normwerte für die CK, Elektrolyte, Transaminasen, Nierenretentionsparameter.

In der arteriellen Blutgasanalyse ergab sich ein $\mathrm{pO}_{2}$ von 75 $\mathrm{mmHg}$, ein $\mathrm{pCO}_{2}$ von $28 \mathrm{mmHg}$, eine Sauerstoffsättigung von $92 \%$ und ein $\mathrm{pH}$ von 7,46 unter Zufuhr von $101 \mathrm{O}_{2} / \mathrm{min}$.

\section{Ergänzende Untersuchungen}

Im EKG zeigte sich ein $\mathrm{S}_{\mathrm{I}} \mathrm{Q}_{\mathrm{II}}$-Lagetyp bei durchgehendem tachykarden Sinusrhythmus mit einer Frequenz von 126/min, zudem fielen in den Brustwandableitungen terminal negative T-Wellen in $\mathrm{V}_{1}-\mathrm{V}_{4}$ auf.

Die Röntgenaufnahme des Thorax zeigte einen Normalbefund, in der abdominellen Sonographie konnte ebenfalls kein pathologischer Befund erhoben werden.
Bei bereits klinisch geäußertem Verdacht auf eine tiefe Beinvenenthrombose wurde eine Duplexsonographie der Beingefäße durchgeführt, in der sich über der rechten V. femoralis superficialis vom Leistenband bis zur V. poplitea keine Flussprofile ableiten ließen, ebenso war hier auf leichten Druck keine Kompression der Vene möglich. Duplexsonographisch wurde die Diagnose einer tiefen Beinvenenthrombose der rechten $\mathrm{V}$. femoralis superficialis vom Leistenband nach distal reichend gestellt. Die kranial des Leistenbands gelegenen Venenabschnitte konnten wegen Darmgasüberlagerung nicht beurteilt werden.

Echokardiographisch fand sich ein massiv erweiterter rechter Ventrikel, bei geringer Trikuspidalinsuffizienz konnte zudem eine pulmonale Hypertonie mit einem systolischen PA-Druck von $54 \mathrm{mmHg}$ über ZVD verzeichnet werden. Ein Embolus ließ sich direkt nicht nachweisen. Der rechte Vorhof war leicht vergrößert, die linksventrikuläre Funktion war unauffällig. Echkardiographisch ergab sich somit der dringende Verdacht auf eine massive Lungenembolie.

Um das Ausmaß der pulmonalen Embolisierung und die Ausdehnung der rechtsseitigen tiefen Beinvenenthrombose nach kranial festzustellen, wurde anschließend eine CT-Untersuchung von Thorax und Becken durchgeführt. Hierbei zeigte sich eine Lungenembolie in den distalen zentralen Anteilen der rechten $A$. pulmonalis und in der rechten Oberlappenarterie (Abb.1). Die Thrombosierung der rechten V. femoralis superficialis ließ sich ab Höhe des Leistenbandes nach kaudal nachweisen.

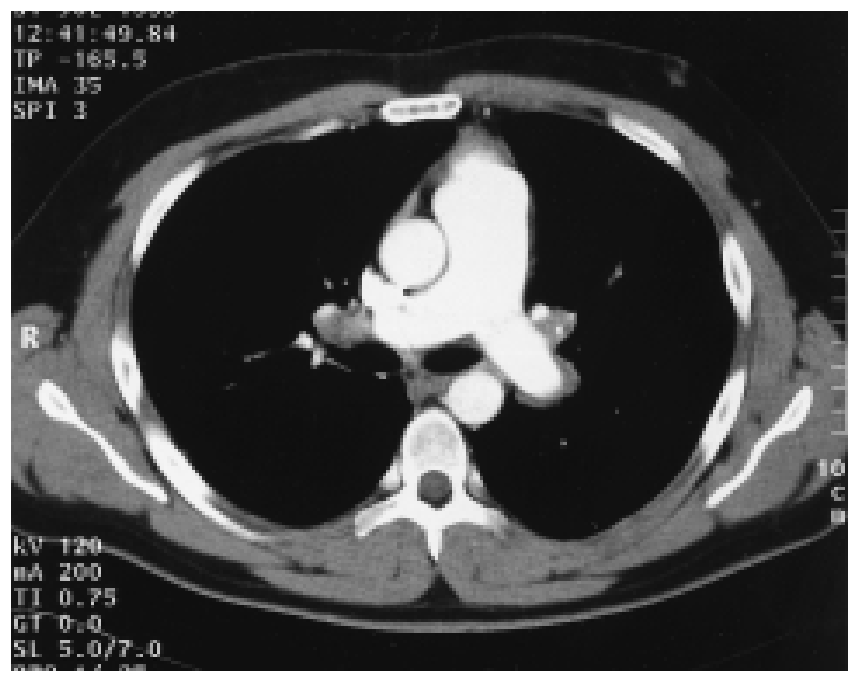

Abb.1 Lungenembolie in den distalen zentralen Anteilen der rechten A. pulmonalis und der rechten Oberlappenarterie.

\section{Therapie und Verlauf}

Bei nachgewiesener rechtsseitiger Lungenembolie und tiefer Beinvenenthrombose wurden im Notfallbereich 5000 E Heparin i.v. verabreicht, es erfolgte dann die Verlegung des Patienten auf die Intensivstation, wo bei fehlenden Kontraindikationen eine systemische Urokinaselyse mit einer Bolusgabe von $200000 \mathrm{E}$ i.v. und anschließend $100000 \mathrm{E} / \mathrm{h}$ eingeleitet wurde. Die Lyse wurde während der folgenden 7 
Tage kontinuierlich durchgeführt. Das Fibrinogen befand sich während der gesamten Zeit im Normbereich (180 - $350 \mathrm{mg} /$ dl). Insgesamt kam es hierunter $\mathrm{zu}$ einer deutlichen klinischen Besserung des Allgemeinzustands des Patienten, subjektiv war eine Besserung der Dyspnoe zu verzeichnen, die initiale Tachykardie ging von $126 / \mathrm{min}$ auf $78 / \mathrm{min}$ zurück, die Sauerstoffsättigung betrug $97 \%$ unter $41 \mathrm{O}_{2} / \mathrm{min}$.

Echokardiographisch kam es zu einem Rückgang des PADrucks systolisch von vormals $54 \mathrm{mmHg}$ auf $18 \mathrm{mmHg}$ über ZVD, der rechte Ventrikel zeigte sich nur geringgradig vergrößert, die Flüsse über allen Herzklappen waren unauffällig.

Zur abschließenden Beurteilung des Lyseergebnisses wurde erneut eine CT-Untersuchung des Thorax durchgeführt. Hierbei zeigte sich im Vergleich zur initialen Voruntersuchung ein deutlicher Befundrückgang der vorher ausgedehnten Lungenembolie mit nur noch wenig nachweisbarem thrombotischen Material in der rechten A. pulmonalis und in der Peripherie der rechten dorsalen Oberlappenarterie (Abb. 2).

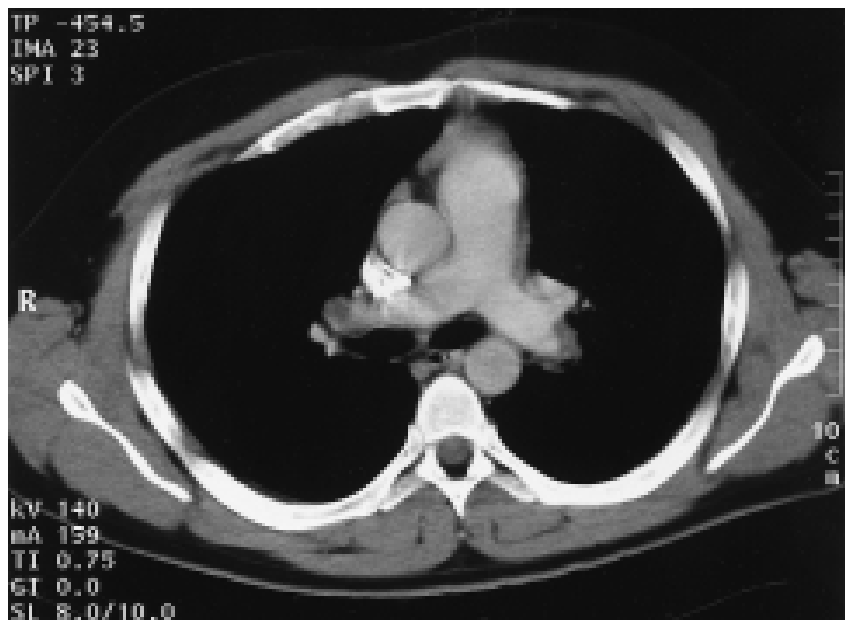

Abb.2 Nach Urokinaselyse über 7 Tage zeigen die zentralen Anteile der rechten A. pulmonalis nur noch wenig thrombotisches Material.

Aufgrund dieses erfreulichen klinischen, echokardiographischen und radiologischen Lyseergebnisses wurde die Urokinasetherapie beendet, es erfolgte im weiteren Verlauf die Umstellung der Antikoagulation auf Heparin i.v., die Verlegung des Patienten auf eine periphere Station und Neueinstellung auf Marcumar. Bei Verlegung zeigte sich laborchemisch eine

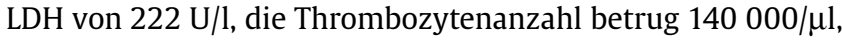
die PTT betrug 96 s unter Heparin 1000 E/h i.v. Die initial zur Thrombophiliediagnostik entnommenen Laborparameter für AT III, Protein C und S, APC-Sensitivität, Faktor V Leiden, Lupus-Antikoagulans, Anti-Cardiolipin-Antikörper, Antiphosphatidylserin-Antikörper, ANA-Screening, Gerinnungsfaktoren und Homocystein zeigten keine pathologischen Werte.

Am 6. Tag nach Verlassen der Intensivstation kam es dann im Laufe des Vormittags nach Toilettengang zu einer plötzlichen massiven Dyspnoe, verbunden mit einer Tachykardie bis 138 Schlägen/min, einer Tachypnoe von 40 Atemzügen/min, die Sauerstoffsättigung betrug nur noch $89 \%$ unter $101 \mathrm{O}_{2} / \mathrm{min}$, der Blutdruck wurde mit 100/50 mmHg gemessen. Es erfolgte daraufhin die erneute Verlegung des Patienten auf die Intensivstation. Im EKG zeigte sich der bereits initial nachgewiesene $S_{I} Q_{I I I}$-Lagetyp mit den bekannten T-Negativierungen über den Vorderwandableitungen. Laborchemisch auffällig waren hierbei eine LDH von $360 \mathrm{U} / \mathrm{l}$, Thrombozytenanzahl von nur

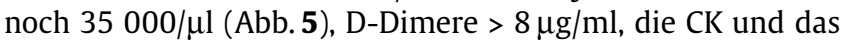
übrige Blutbild waren unauffällig, die PTT betrug $90 \mathrm{~s}$, der Quick-Wert lag bei 78\%, die Umstellung auf Marcumar war erst 2 Tage zuvor begonnen worden. Echokardiographisch zeigte sich ein deutlich dilatierter rechter Ventrikel mit paradoxer Septumbewegung und einer nachweisbaren Trikuspidalinsuffizienz mit einem maximalen Gradienten über der Trikuspidalklappe von $60 \mathrm{mmHg}$ und einer zusätzlichen Dilatation des rechten Vorhofs. Im Thorax-CT ergaben sich ausgedehnte zentrale Lungenembolien beidseits, die Pulmonalarterienhauptstämme waren ursprungsnah durch kugelige Thromben verschlossen, der Truncus pulmonalis und der rechte Ventrikel massiv dilatiert (Abb.3). Eine Restperfusion zeigte sich noch im Bereich der Lingula und des Oberlappens linksseitig sowie eine teilweise Minderperfusion in den Unterlappen beidseits. Im Becken-CT war eine Zunahme der vormals nachgewiesenen Beinvenenthrombose nachweisbar, ein im Bereich der V. femoralis und V. saphena magna umspülter Thrombuszapfen reichte nach kranial in die V. iliaca externa und endete in Höhe der Mündung der V. iliaca interna, das Confluens venosum und die Vv. iliacae communes waren beidseits frei.

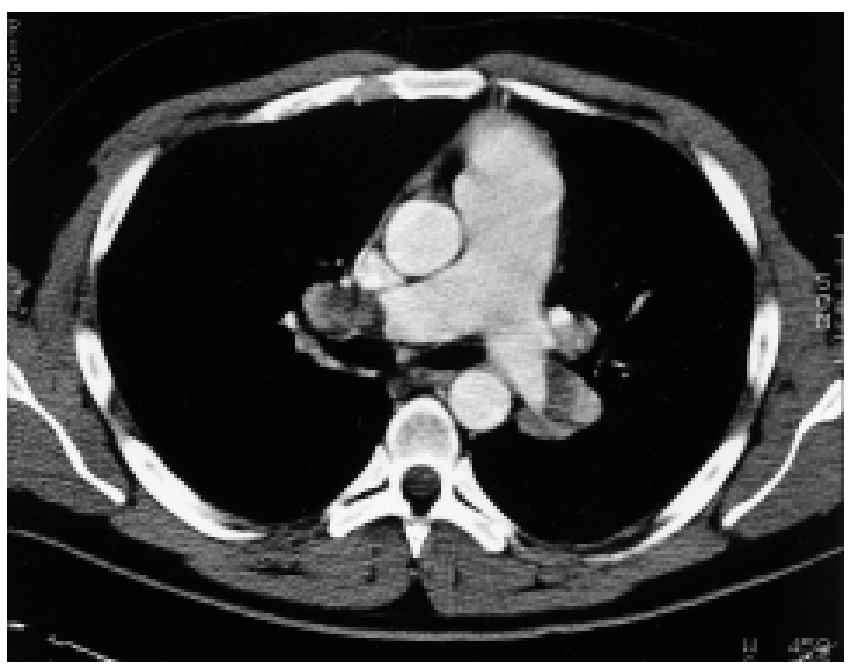

Abb.3 Ausgedehnte zentrale Rezidivlungenembolien beidseits, die beiden Pulmonalarterienhauptstämme sind durch kugelige Thromben verschlossen, der Truncus pulmonalis ist deutlich dilatiert.

Verbunden mit dem klinischen Befund eines massiven Lungenembolierezidivs und der neu aufgetretenen Thrombozyto-

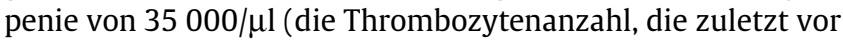

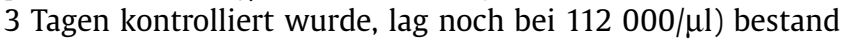
der dringende klinische Verdacht auf eine heparininduzierte Thrombozytopenie Typ II (HIT II). Mittels ELISA konnte der positive Nachweis von Antikörpern gegen Plättchenfaktor 4 (PF4)-Heparin-Komplexe noch am gleichen Tag erbracht werden (Test der Firma Genetic Testing Institute, Brookfield, Wyoming, USA). Ein HIPA-Test (heparininduzierte Plättchen- 
aktivierung) wurde aufgrund der eindeutigen klinischen Symptomatik nicht durchgeführt.

Es erfolgte das sofortige Absetzen der systemischen Heparinisierung und die Umstellung der Antikoagulation auf Lepirudin $\left(\right.$ Refludan $^{\circledR}$ ) i.v., die Steuerung erfolgte über die aPTT (initial $0,4 \mathrm{mg} / \mathrm{kg}$ iv. als Bolus, danach $0,15 \mathrm{mg} / \mathrm{kg} / \mathrm{h}$ als Dauerinfusion verabreicht). Am gleichen Tag wurde dem Patienten ein temporärer Günther-Tulip-V.-cava-Filter zwischen der Mündung der Nierenvenen und der distalen Aufzweigung der V. cava inferior in die Iliakalvenen eingesetzt. Unter der Lepirudintherapie kam klinisch es zu einem deutlichen Rückgang der Tachykardie von 138 auf 105/min und zu einem Anstieg der Sauerstoffsättigung auf $96 \%$ unter $81 \mathrm{O}_{2} / \mathrm{min}$. Laborchemisch kam es auch zu einem sukzessiven Anstieg der Thrombozytenzahl von $35000 / \mu$ l auf $190000 / \mu l$ innerhalb dieser 3 Tage. Nach Anstieg der Thrombozytenzahl wurde eine erneute systemische Urokinaselyse eingeleitet, die für weitere 10 Tage durchgehend fortgeführt wurde. Die Lepirudintherapie wurde während dieser Zeit pausiert. Echokardiographisch kam es darunter im Verlauf zu einem Rückgang des Gradienten über der Trikuspidalklappe auf $32 \mathrm{mmHg}$. Nach Beendigung der Urokinaselyse fand sich im thorakalen CT insgesamt ein deutlicher Befundrückgang der vormals ausgedehnten zentralen Lungenembolien beidseits, jedoch war der Thrombus im Hauptstamm der rechten A. pulmonalis noch fast unverändert darstellbar (Abb.4). Auch im CT des Beckens war ein Rückgang der Beinvenenthrombosierung bis zu den distalen Anteilen der V. iliaca externa bei jedoch unveränderter Thrombosierung der V. femoralis superficialis nachweisbar.

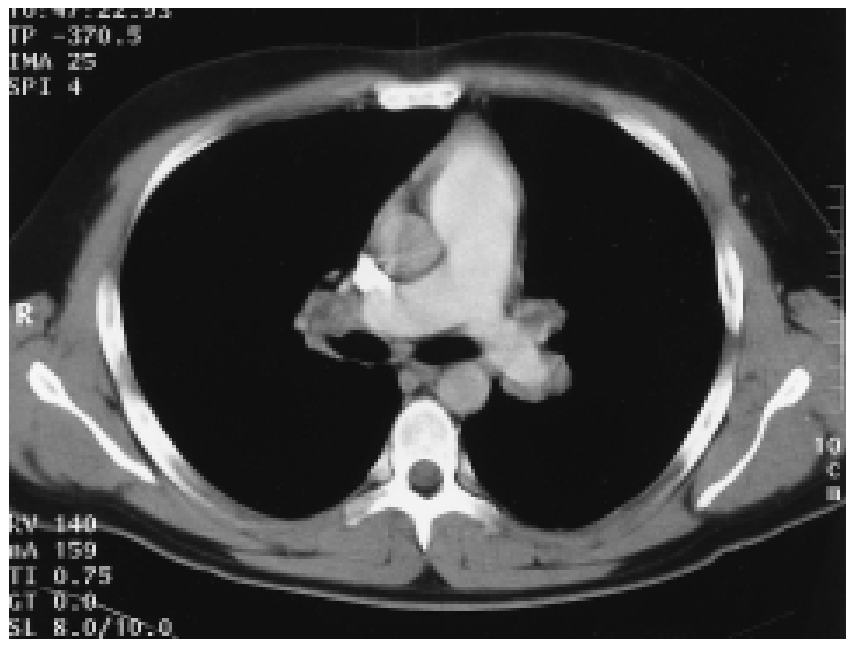

Abb.4 Nach erneuter Urokinaselyse über 10 Tage deutlicher Befundrückgang der vormals ausgedehnten beidseitigen zentralen Lungenembolie, jedoch noch Nachweis eines Thrombus im rechten Pulmonalishauptstamm.

Nach Urokinaselyse wurde der Patient weiterhin mit Lepirudin antikoaguliert und der temporäre V.-cava-Filter entfernt. Über die noch liegende zentrale Schleuse wurden mittels eines pulmonalen Einschwemmkatheters die PA-Drücke mit 45/22 mmHg und die rechtsventrikulären Drücke mit 42/10 $\mathrm{mmHg}$ gemessen. Eine Kontrolluntersuchung ist nach Ablauf von 2 Monaten vorgesehen.

\section{Thrombozytenverlauf}

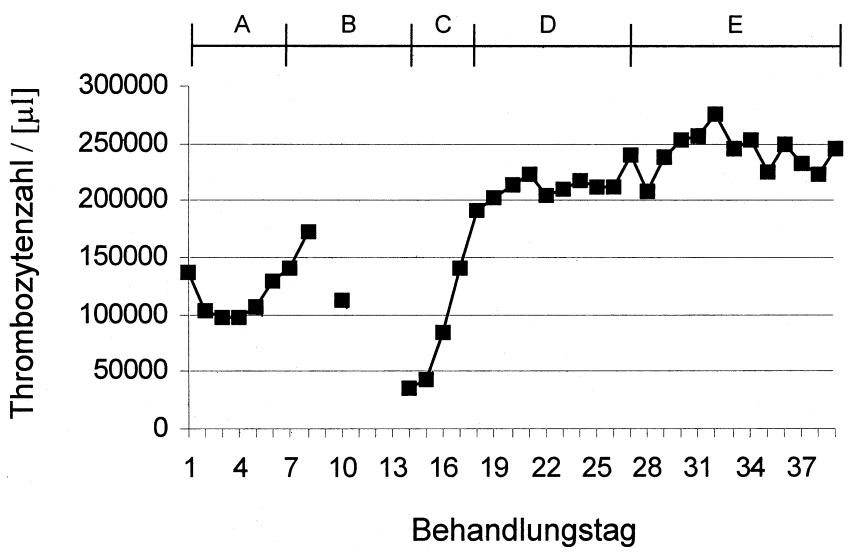

Abb.5 Thrombozytenverlauf während der 1. Urokinaselyse (A), systemischer Heparinisierung (B), Umstellung der Antikoagulation auf Lepirudin (C), 2. Urokinaselyse (D) und anschließender Marcumarisierung unter protektiver erneuter Lepirudintherapie (E).

Nach Umstellung auf Marcumar konnte der Patient 9 Tage nach Verlegung auf Normalstation im guten Allgemeinbefinden entlassen werden. Ein Notfallausweis, der den Patienten bei erneuter Heparinexposition als HIT-II-gefährdet ausweist und den er lebenslang bei sich tragen muss, wurde dem Patienten ausgestellt.

\section{Diskussion}

Heparin und niedermolekulare Heparine gehören $\mathrm{zu}$ den wichtigsten und am häufigsten verwendeten Medikamenten zur Prophylaxe von Gefäßverschlüssen im stationären und ambulanten Bereich. Durch ihre breite und regelmäßige Anwendung gehört die heparininduzierte Thrombozytopenie (HIT), als eine der bedeutendsten und vital gefährlichsten Komplikationen unter Heparintherapie, zu den häufigsten medikamenteninduzierten Thrombozytopenien.

Die heparininduzierte Thrombozytopenie Typ I (HIT I) tritt bei ca. $10 \%$ der Patienten unter Heparintherapie auf, dabei fallen die Thrombozyten meistens innerhalb der ersten 5 Tagen nach Beginn der Heparingabe ab. Ein Wert von $100000 / \mu \mathrm{l}$ wird hierbei selten unterschritten (Abnahme um höchstens 30\% vom Ausgangswert), die Thrombozytenzahlen steigen trotz weiterer Heparintherapie wieder auf Normalwerte an. Komplikationen im Sinne von Blutungen oder Thromboembolien sind nicht zu erwarten [1]. Pathophysiologisch handelt es sich hierbei um einen nichtimmunologischen, durch Heparin direkt an den Thrombozyten ausgelösten proaggregatorischen Effekt. Die Bindung von Heparin an verschiedene Proteine der Thrombozytenoberfläche (z. B. Plättchenfaktor 4 [PF 4]) führt zur Senkung des thrombozytären cAMP, wodurch die Aktivierungsschwelle der Thrombozyten sinkt. Die Bindung des Heparins an die Plättchenoberfläche ist u.a. abhängig vom Sulfatierungsgrad und Molekulargewicht des verwendeten Heparins (je längerkettig das Heparin und je mehr Sulfatgruppen es trägt, desto stärker die Bindung an die Thrombozyten und um so stärker der proaggregatorische Effekt) [1,2]. Die HIT I erfordert in der 
Regel keine Behandlung. Eventuell kann die Gabe eines LMWHeparins sinnvoll sein, da Molekulargewicht und Sulfatierungsgrad bei dieser Heparingruppe niedriger sind und damit auch der proaggregatorische Effekt. Sollte die Heparintherapie weitergeführt werden, so ist die tägliche Kontrolle der Thrombozyten unerlässlich, da die Entwicklung einer HIT II möglich ist.

Die HIT II stellt mit einer Inzidenz von 0,5 - 3\% eine seltene, jedoch potenziell lebensgefährliche Komplikation unter Heparintherapie dar $[1,3,4]$. Sie tritt typischerweise $5-14$ Tage nach Beginn der Heparingabe auf, selten erst nach der 3. - 4. Woche. Bei jedem Abfall der Thrombozyten um mehr als 50\% gegenüber dem höchsten Wert ab dem 5. Tag unter HeparinTherapie muss die HIT II differenzialdiagnostisch beachtet werden. Der Thrombozytenabfall ist in den meisten Fällen das Leitsymptom, sie liegen häufig zwischen 40000 und 60 000/ $\mu \mathrm{l}$. Nach Absetzen des Heparins kommt es meistens zu einer Normalisierung der Thrombozytenwerte innerhalb von 3-7 Tagen $[1,3,5]$.

Neben dem Leitsymptom der Thrombozytopenie fallen die Patienten durch neu auftretende venöse und arterielle Gefäßverschlüsse auf und sind vital gefährdet. Von den arteriellen Gefäßverschlüssen, die seltener als die venösen beobachtet werden, sind vor allem die großen Arterien der Extremitäten, die Koronargefäße und Hirnarterien betroffen. Sowohl die Thrombozytopenie als auch die thromboembolischen Komplikationen sind dabei unabhängig von der Menge, der Verabreichungsart (i.v., s.c. oder i.m.) und der Heparinart (fraktioniert oder unfraktioniert) [2]. Selbst kleinste Mengen Heparin aus heparinbeschichteten intraarteriellen Kathetern können eine HIT II auslösen. Bei Patienten, welche schon einmal Heparin erhalten haben, kann die HIT II jedoch bereits innerhalb weniger Stunden nach Reexposition manifest werden und nicht erst nach der oben erwähnten Latenz von 5 Tagen nach Beginn der Heparintherapie [4].

Pathophysiologisch handelt es sich bei der HIT II um einen immunologischen Mechanismus, für den auch das Zeitintervall von 5-14 Tagen zwischen Beginn der Heparinexposition und Manifestwerden der HIT II spricht. Es handelt sich dabei um eine durch Heparin-, PF4- und IgG-vermittelten Aktivierung von Thrombozyten im Plasma und am Gefäßendothel und einer dadurch bedingten Thrombozytenaggregation (die gegen PF4-Heparin-Komplexe gerichteten Antikörper binden an die gleichen Epitope auf Thrombozyten und Endothelzellen) [6]. Die Gefäßverschlüsse bestehen aus Thrombozyten, Fibrin, einer geringen Anzahl von Leukozyten, jedoch nur wenigen Erythrozyten („white clot syndrome“), dies deutet auch auf einen peripheren „Verbrauch“ der Thrombozyten als Ursache der Thrombozytopenie.

Die klinische Manifestation der HIT II ist somit von mehreren Faktoren abhängig: dem Molekulargewicht und Sulfatierungsgrad des verwendeten Heparins und damit seiner proaggregatorischen Wirkung (siehe oben), dem Zustand des Immunsystems des Patienten, der Länge der Antigenexposition (Zeitdauer der Heparingabe) und der Voraktivierung der Thrombozyten (Patienten mit manifesten Thrombosen haben bereits voraktivierte Thrombozyten!).
Zu den Diagnosekriterien der HIT II gehören neben dem Leitsymptom der Thrombozytopenie 5-14 Tage nach Heparingabe und dem Neuauftreten thromboembolischer Komplikationen, eine normale Thrombozytenzahl vor Beginn der Heparintherapie, ein Wiederanstieg der Thrombozyten nach Absetzen des Heparins, der Ausschluss anderer Ursachen einer Thrombozytopenie (z. B. Sepsis, Pseudothrombozytopenie, Verbrauchskoagulopathie, Autoimmunthrombozytopenie usw.) und laborchemische Tests. $\mathrm{Zu}$ den laborchemischen Nachweisverfahren gehören der HIT-II-Test mittels ELISA, bei dem Antikörper gegen PF4-Heparin-Komplexe nachgewiesen werden und der HIPA-Test (heparininduzierte Plättchenaktivierung), ein Funktionstest unter Verwendung gewaschener Thrombozyten [5].

Die wichtigste therapeutische Maßnahme bei nachgewiesener HIT II ist das sofortige Absetzen des Heparins. Bei aufgetretenen Thrombosen kann die Einleitung einer Lyse mit rtPA, Streptokinase oder Urokinase bei fehlenden Kontraindikationen erwogen werden. Eine Gabe von Thrombozytenkonzentraten ist kontraindiziert, da sie die Bildung neuer Gefäßverschlüsse begünstigen. Patienten mit nachgewiesener HIT II dürfen im Laufe ihres Lebens nicht wieder Heparin gegenüber exponiert werden. Bei erneuter Exposition mit einem inkompatiblen Heparin kann es hierbei aufgrund der Vorsensibilisierung bereits innerhalb von Stunden zum Auftreten thromboembolischer Komplikationen kommen, die den Patienten vital gefährden können. Die intensive Aufklärung des Patienten und die Ausstellung eines Notfallausweises sind deshalb unerlässlich. Heparin ist $\mathrm{zu}$ vermeiden bei Katheterspülungen, Hämodialyse, Hämofiltration und sollte hierbei durch ein geeignetes Antikoagulans (siehe unten) ersetzt werden [7,8]. Ferner sollte beachtet werden, dass PPSB (Prothrombinkomplex) und andere Gerinnungspräparate Heparin enthalten.

Zur Fortführung der Antikoagulation sind derzeit ein Heparinoid, das Danaparoid $\left(\operatorname{Orgaran}^{\circledR}\right.$ ), das seit 1983 mit Erfolg bei der HIT II eingesetzt wird, und rekombinantes Hirudin zugelassen.

Hirudin, ein Einketten-Polypeptid aus 65 Aminosäuren, ist ein direkt wirkender spezifischer Inhibitor von Thrombin. Darüber hinaus hemmt Hirudin alle bisher bekannten Interaktionen von Thrombin mit zellständigen Rezeptoren, z. B. Endothelzellen [9]. Seine Wirkung ist AT-III-unabhängig, ferner wird Hirudin nicht durch PF4 gehemmt. Hirudin ist antigen wirksam, es besteht jedoch keine Kreuzreaktivität zu HIT-II-Antikörpern. Diese ist in 10\% der Fälle bei Verwendung von Danaparoid zu beobachten. Die Steuerung der Antikoagulation erfolgt über die aPTT. Hirudin wird in der Niere glomerulär filtriert und durch Proteasen gespalten, bei Niereninsuffizienz ist deshalb die Halbwertszeit von Hirudin (normalerweise ca. 3 Stunden) verlängert und eine entsprechende Dosisreduktion erforderlich. Bei Überdosierungen ist die Inzidenz von Blutungen unter Hirudin höher als unter Heparin, eine Kontrolle der aPTT sollte deshalb in der Anfangsphase alle 4 Stunden erfolgen. Die Dosierung von Lepirudin erfolgt mit $0,4 \mathrm{mg} / \mathrm{kg}$ KG als Bolusinjektion i.v., anschließend erfolgt eine Dauerinfusion mit $0,15 \mathrm{mg} / \mathrm{kg} / \mathrm{h}$, wobei hier eine engmaschige Kontrolle der aPTT wie oben erwähnt erfolgen sollte. Bei Patienten mit nachgewiesener 
HIT II und thromboembolischen Komplikationen sollte die aPTT das 1,5 - 3fache des Ausgangswertes betragen [8].

Im Handel sind Desirudin (Revasc ${ }^{\circledR}$ ) und Lepirudin (Reflu$\operatorname{dan}^{\circledR}$ ) zur Antikoagulation bei nachgewiesener HIT II erhältlich. Desirudin ist zur Prophylaxe einer tiefen Beinvenenthrombose bei Patienten nach Hüft- und Kniegelenksendoprothesen-Operation zugelassen (Dosierung: $2 \times 15 \mathrm{mg} / \mathrm{d}$ subkutan) [8].

Im geschilderten Fall erfolgte die stationäre Aufnahme des Patienten aufgrund einer rechtsseitigen Lungenembolie bei tiefer Beinvenenthrombose. Nach Ausschluss anderer Thromboseursachen ist die Thrombose der V. femoralis superficialis am ehesten durch die venöse Stase im Zusammenhang mit den in der Woche davor durchgeführten längeren Autofahrten zu sehen. Der initial registrierte Thrombozytenwert betrug

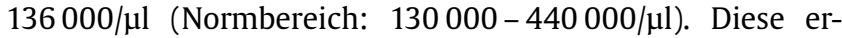
niedrigte, bei Aufnahme gemessene Thrombozytenanzahl ist am ehesten als Ausdruck eines peripheren „Verbrauchs“ der Thrombozyten im Bereich der ausgedehnten Beinvenenthrombose und der Lungenembolie $\mathrm{zu}$ werten. Im weiteren

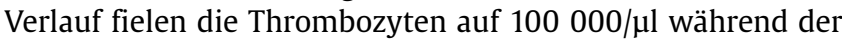
ersten 3 Tage der Lysetherapie ab. Da der Patient in der Aufnahmesituation bereits Heparin erhalten hatte, mag dieser erste Thrombozytenabfall retrospektiv bereits als Ausdruck einer beginnenden HIT II zu interpretieren sein. Unter der Lysetherapie war es zu keinen zusätzlichen thromboembolischen Komplikationen gekommen und die Thrombozytenwerte stiegen wieder auf Normalwerte an. Unter der nachfolgenden Urokinaselyse über 7 Tage besserte sich die klinische Symptomatik zusehends, auch radiologisch war eine Größenregredienz der pulmonalen Emboli zu verzeichnen. Während der darauf folgenden Woche war der Patient durchgehend systemisch vollheparinisiert gewesen, die Thrombozytenanzahl wurde jedoch nicht täglich kontrolliert. Am erneuten Aufnahmetag auf der Intensivstation war die Klinik gekennzeichnet durch ein massives, hämodynamisch wirksames zentrales Lungenembolierezidiv beidseits und einer Zunahme der rechtsseitigen Beinvenenthrombose bei manifester HIT II. Die Thrombozyten des Patienten waren durch die vorhergehende Lungenembolie und die Beinvenenthrombose bereits voraktiviert, so dass der proaggregatorische Effekt bei der HIT II verstärkt ablaufen konnte (siehe oben). Die Thrombozytenzahl betrug $35000 / \mu \mathrm{l}$, der zuletzt vor 3 Tagen kontrollierte Thrombozytenwert wurde mit 112 000/ $\mu$ l gemessen. Unter der Umstellung auf Lepirudin konnte schon allein ein Rückgang der Tachykardie und Tachypnoe beobachtet werden, das vermutlich auf dessen Antithrombinwirkung zurückzuführen ist. Die Sauerstoffsättigung betrug jedoch nur 96\% unter 81 $\mathrm{O}_{2} /$ min, unter Raumluft fiel sie auf $88-90 \%$ herab, weswegen wir uns bei dem nur leidlich hämodynamisch stabilen jungen Patienten zu einer erneuten Lysetherapie entschlossen. Die Lysetherapie wurde zunächst für 5 Tage durchgeführt, da jedoch eine nach 5 Tagen Lysetherapie veranlasste CT-Untersuchung (hier als Abbildung nicht aufgeführt) keine wesentliche Auflösung der pulmonalen Emboli zeigte und der Patient auch klinisch bisher nicht ausreichend profitierte, entschlossen wir uns zur Weiterführung der Urokinaselyse für weitere 5 Tage. Erfreulicherweise konnte diese zweite Phase der Lysetherapie zu einer weitgehenden Auflösung der neu aufgetretenen pulmonalen Emboli führen, der radiologische End- befund ist in etwa vergleichbar mit dem nach Beendigung der ersten Lysetherapie.

Da derzeit noch wenige Erfahrungen über eine Lysetherapie bei HIT II und über die Kombination von Lepirudin mit einem Thrombolytikum vorliegen, wurde in unserem Fall die Lepirudingabe während der Urokinaselyse nicht fortgesetzt. Vereinzelt wurde über eine erfolgreiche Lyse bei arteriellen und venösen Thrombosen berichtet, wobei hier sowohl die Dosierung des Lepirudin als auch die des Thrombolytikums, je nach lokaler oder systemischer Lysetherapie, jeweils der klinischen Situation angepasst wurde $[10,11,12]$. Von Seiten des Herstellers wird wegen der mangelnden Erfahrung eine „drastische“ Dosisreduktion ohne konkrete Mengenangaben empfohlen (Fachinformation Refludan ${ }^{\circledR}$, Stand 1997). Neuere Literaturquellen empfehlen bei Patienten mit HIT II unter gleichzeitiger Thrombolyse eine Bolusgabe von $0,2 \mathrm{mg} / \mathrm{kg}$ i.v. und anschließend eine Dauerinfusion mit $0,1 \mathrm{mg} / \mathrm{kg} / \mathrm{h}$, wobei die aPTT das 1,5-3fache des Ausgangswerts betragen soll [8]. Es wird ausdrücklich betont, dass die Dosierungsangaben als Empfehlungen und nicht als Absolutwerte zu verstehen sind, die Dosierung muss im Einzelfall angepasst werden. Studiendaten zur Lysetherapie bei HIT-Patienten liegen derzeit leider noch nicht vor.

Schlussfolgernd sollten bei jedem Patienten unter Heparintherapie, vor allem wenn Heparin bei bereits manifesten Thrombosen eingesetzt wird, die Thrombozyten am Anfang und ab dem 5. Tag der Heparingabe jeden 2. Tag, ab dem 20. Tag einmal pro Woche kontrolliert werden. Der Thrombozytenabfall sollte rechtzeitig vor dem Auftreten thromboembolischer Komplikationen erkannt werden, um die entsprechende Umstellung der Antikoagulation vorzunehmen. Es sei darauf hingewiesen, dass sich in ca. 30\% der Fälle die Thrombozytopenie erst nach Auftreten eines Gefäßverschlusses manifestiert, so dass die tägliche klinische Untersuchung des Patienten unentbehrlich bleibt.

\section{Literatur}

${ }^{1}$ Greinacher A. Heparin-induzierte Thrombozytopenie. Internist 1996; 37: $1172-1178$

${ }^{2}$ Warkentin TE, Levine NW, Hirsh J, Horsewood P, Roberts RS, Tech M, Gent M, Kelton JG. Heparin-induced thrombocytopenia in patients treated with low molecular weight or unfractionated heparin. New Engl J Med 1995; 332: 1330-1334

${ }^{3}$ Eder $S$ et al. Heparin-induzierte Thrombozytopenie Typ II. Chirurg 1999; 70 (10): 1149-1155

${ }^{4}$ Langer M. Heparin-induzierte Thrombozytopenie. Dtsch Med Wochenschr 1999; 124 (40): 1189

${ }^{5}$ Greinacher A, Müller-Eckhardt C. Diagnostik der Heparin-assoziierten Thrombozytopenie. Dtsch Med Wochenschr 1991; 116: $1479-1482$

${ }^{6}$ Greinacher A, Pötzsch B, Amiral J et al. Heparin-associated thrombocytopenia: isolation of the antibody and characterisation of a multimolecular PF4-heparin complex as the major antigen. Thromb Haemost 1994; 71: $247-251$

${ }^{7}$ Fischer KG. Recombinant hirudin (lepirudin) as anticoagulant in intensive care patients treated with continous hemodialysis. Kidney Int Suppl 1999; 72: $41-45$

${ }^{8}$ Ranze O, Greinacher A. Aktuelle Behandlungskonzepte bei Heparin-induzierter Thrombozytopenie. Dtsch Med Wochenschr 1999; $124: 865-873$ 
${ }^{9}$ Nowak G. Hirudin: Pharmakologie und Therapie. In: MüllerBerghaus G, Pötzsch, B (ed) Hämostaseologie. Berlin Heidelberg New York; Springer, 1999: 698-703

${ }^{10}$ Emig U, Meyer T, Buchwald AB. Erfolgreiche Lysetherapie einer Vena-cava-inferior und iliofemoral- Venenthrombose bei Heparin-induzierter Thrombozytopenie Typ II. Dtsch Med Wochenschr 1997; 122: 965 -968

${ }^{11}$ Olbrich K, Wiersbitzky M, Wacke W, Eichler P, Zinke H, Schwock M, Möx B, Kraatz G, Motz W, Greinacher A. Atypical heparininduced thrombocytopenia complicated by intracardiac thrombus, effectively treated with ultra low dose rtPA lysis and recombinant hirudin (lepirudin). Blood. Coagul. Fibrinolysis 1998; 9: 273-277

12 Weidmann B, Gerber V, Rinas U, Jansen W, Tauchert M. Behandlung einer schweren Lungenembolie bei Heparin-induzierter Thrombozytopenie Typ II (HIT II) mit Alteplase und Lepirudin. Intensivmed 1999; 36: 449-453

\section{Dr. F. Nickels}

Klinik und Poliklinik für Innere Medizin II Klinikum der Universität Regensburg

Franz-Josef-Strauß-Allee 11

93042 Regensburg 